Ann. Biol. anim. Bioch. Biophys., I962, 2 (2), I63-166

\title{
ACTION DE L'ATROPINE SUR LE COMPORTEMENT SEXUEL ET LA COMPOSITION DU SPERME CHEZ LE TAUREAU
}

\author{
J. P. SIGNORET \\ Avec la collaboration technique de P. Evrard \\ Station de Recherches de Physiologie animale, \\ Centre national de Recherches zootechniques, Jouy-en-Josas (Seine-et-Oise).
}

\section{SOMMAIRE}

I3 taureaux ont reçu 3 o minutes avant la collecte de sperme au vagin artificiel une injection sous-cutanée, soit de $800 \mathrm{mg}$ d'atropine, soit de sérum physiologique. Les animaux présentent après l'injection d'atropine des réactions nettes d'énervement, de météorisation, mais leur comportement sexuel n'est pas perturbé.

Les caractéristiques du sperme obtenu sont nettement modifiées, après l'injection d'atropine : le volume passe de $6, \mathrm{I} \mathrm{ml}$ à $4,0 \mathrm{ml}$, la concentration de I 349000 à I 613000 spermatozoïdes $/ \mathrm{mm}^{3}$ et le nombre total de 8,4 milliards à 6,6 milliards.

L'action d'une injection sur la composition de l'éjaculat est encore sensible après 3 ou 4 jours, alors que toute réaction extérieure a disparu au bout de 24 heures.

Ces résultats sont susceptibles d'être utilisés pour une analyse des mécanismes de l'éjaculation.

L'action de l'atropine sur la composition du sperme a été mise en évidence chez le Verrat par Dziuk (I959) : alors qu'aucun effet n'est constaté sur le comportement sexuel, le volume de sperme obtenu par éjaculation devient égal à 16 p. Ioo de celui des témoins, la concentration en spermatozoïdes est près de cinq fois plus forte et le nombre total de spermatozoïdes reste pratiquement inchangé.

Cette action, permettant de diminuer la proportion des sécrétions accessoires dans l'éjaculat, présente un grand intérêt aussi bien en ce qui concerne l'étude théorique de l'éjaculation que pour l'application pratique à l'insémination artificielle.

Nous avons donc entrepris chez le Taureau une étude analogue dont les premiers résultats sont rapportés ici.

\section{MATÉRIEL, ET MÉTHODES}

Nous avons utilisé I 3 taureaux, dont cinq couples de jumeaux, appartenant aux trois plus importantes races laitières française : Normande, Frisonne et Pie rouge de l'Est, et dont les poids s'échelonnent entre 700 et $900 \mathrm{~kg}$ et les âges entre 2 et 6 ans 
Deux fois par semaine un éjaculat a été récolté sur chaque taureau et les contrôles suivants effectués :

- motilité des spermatozoïdes notée subjectivement de o à 4, selon une échelle utilisée dans la routine du laboratoire,

- volume mesuré au i/ro de $\mathrm{ml}$,

- concentration en spermatozoïdes déterminée par néphélométrie,

- taux de spermatozoïdes morts déterminé par coloration différentielle à l'éosine-nigrosine (BLom, 1950).

La collecte du sperme était faite au vagin artificiel après un délai d'excitation de 5 minutes pendant lequell le taureau effectuait un chevauchement sans éjaculation.

La solution de sulfate d'atropine à 2 p. 100 dans du sérum physiologique était injectée par voie sous-cutanée. La dose utilisée, très supérieure aux doses thérapeutiques usuelles, a été après divers essais fixée à $800 \mathrm{mg}$ par animal, soit en moyenne I mg par kilogramme de poids vif. Les témoins recevaient une injection équivalente de sérum physiologique stérile.

Le délai entre l'injection et le début de la période d'excitation précédant la collecte a été fixé à 30 minutes.

L'expérience a été divisée en trois périodes de quinze jours séparées chacune par un mois de repos. Au cours de chaque période les animaux étaient répartis en deux groupes.

I $^{0}$ Un groupe expérimental recevant avant une collecte sur deux une injection d'atropine et avant la suivante une injection de sérum physiologique, afin d'éviter un effet cumulatif de l'atropine.

$2^{\circ}$ Un groupe témoin recevant une injection de sérum physiologique avant chaque collecte.

Une permutation circulaire des traitements était effectuée au cours des périodes expérimentales successives.

\section{RÉSULTATS ETT DISCUSSION}

Les réactions des animaux à ces doses relativement fortes d'atropine sont nettes. L'animal bouge sans cesse, est inquiet, ne mange pas et présente souvent un début de météorisation, ces symptômes disparaissent cependant dès le lendemain de l'injection sans laisser de séquelles.

Le comportement sexuel des animaux n'a en rien été perturbé. Comme les témoins, tous les animaux traités se sont montrés très ardents, chevauchant le bouteen-train dès que la possibilité leur en était laissée, sans latence appréciable. L'éjaculation a été obtenue aussi facilement que chez les témoins.

Les caractéristiques du sperme récolté sont rassemblées dans le tableau I.

TABLEAU I

Action de l'atropine sur les caractéristiques des éjaculats de Taureau

\begin{tabular}{|c|c|c|c|c|c|c|c|}
\hline & $\begin{array}{c}\text { Dose } \\
\text { Atropine }\end{array}$ & $\begin{array}{c}\text { Nombre } \\
\text { de } \\
\text { Collectes }\end{array}$ & $\begin{array}{c}\text { Volume } \\
\text { en ml }\end{array}$ & $\begin{array}{l}\text { Motilité } \\
\text { de } 0 \text { d' } 4\end{array}$ & $\begin{array}{c}\text { Concentration } \\
\times 10^{3} \\
\text { par } \mathrm{mm}\end{array}$ & $\mid \begin{array}{l}\text { Nombre total } \\
\text { de spz. } \times 10^{6}\end{array}$ & $\begin{array}{c}\text { Nombre total } \\
\text { de spz. } \\
\text { vivants } \times 10^{\circ}\end{array}$ \\
\hline \multirow{2}{*}{$\begin{array}{l}\text { Groupe } \\
\text { expérimental }\end{array}$} & $800 \mathrm{mg}$ & 58 & $4,03 \pm 0,25$ & $2,75 \pm 0,09$ & $1613 \pm 66$ & $6580 \pm 533$ & $5901 \pm 464$ \\
\hline & 0 & 48 & $5,23 \pm 0,35$ & $2,9^{\prime} \pm 0,09$ & $1470 \pm 85$ & $7422 \pm 613$ & $6640 \pm 582$ \\
\hline Groupe témoin & 0 & 49 & $6,10 \pm 0,37$ & $2,81 \pm 0,09$ & 1349 上 79 & $8401 \pm 716$ & $7455 \pm 649$ \\
\hline
\end{tabular}

En comparant les éjaculats des animaux soumis à l'injection d'atropine à ceux des témoins, on note une très forte réduction du volume du sperme $(\mathrm{P}<0, \mathrm{OOI})$, une augmentation de la concentration en spermatozoïdes $(P<0,02)$ mais une diminution du nombre total de spermatozoïdes $(\mathrm{P}=0,05)$. 
Les caractéristiques des éjaculats des animaux soumis à une injection de sérum physiologique, mais ayant reçu de l'atropine 3 ou 4 jours avant, ont des valeurs intermédiaires. Néanmoins aucune différence n'est significative.

$L_{1}$ taux de spermatozoïdes morts et la motilité ne sont en aucun cas modifiés.

\section{Discussion.}

Au cours de ce travail, aucune action sur le comportement sexuel n'a été enregistrée malgré les doses importantes utilisées. Ceci est en accord avec les résultats de DzIUK ( (959) chez le Verrat, mais est en contradiction avec ceux obtenus chez le Rat par SoulaIRaC (I957). Cet auteur observe en effet une disparition complète de tout comportement sexuel dans les 20 minutes suivant l'injection. Il est à remarquer qu'il utilise cependant des doses proportionnellement beaucoup plus fortes

En ce qui concerne l'action sur la composition du sperme nos résultats sont comparables à ceux obtenus par DzıuK chez le Verrat ; cependant la plus faible proportion des sécrétions accessoires dans l'éjaculat des bovins explique l'action moins nette, quoique significative, sur le volume et la concentration.

L'injection d'atropine semble donc réduire, dans l'éjaculat, la part occupée par les sécrétions des glandes annexes; le mécanisme d'émission des spermatozoïdes ne serait que peu touché puisque, à la diminution du volume de l'éjaculat, correspond une augmentation de la concentration en spermatozoïdes.

Trois hypothèses peuvent être émises quant à cette action sur les sécrétions accessoires :

- Soit un blocage du système parasympathique : la contraction de certaines glandes à sécrétions accessoires serait provoquée par l'action du parasympathique, alors que l'émission des spermatozoïdes et l'éjaculation dépendraient d'un mécanisme différent.

- Soit un blocage de la sécrétion d'une ou plusieurs formations glandulaires du tractus génital, à l'image de celui que l'atropine provoque sur la sécrétion des glandes salivaires.

- Soit le blocage d'un éventuel nerf excito-secréteur parasympathique

L'action résiduelle observée 3 ou 4 jours après l'injection d'atropine tendrait à étayer cette deuxième hypothèse ; par contre, la rapidité de l'action, 30 minutes après une injection sous cutanée, laisserait plutôt supposer un blocage du parasympathique.

Nous nous proposons au cours d'expériences ultérieures d'analyser plus complètement ces phénomènes. L'action de l'atropine sur la composition du sperme du Taureau semble en effet assez nette pour que cette étude puisse apporter des renseignements intéressants sur le mécanisme de l'éjaculation.

Rę̧u en janvier 1962.

\section{SUMMARY}

\section{ACTION OF ATROPINE ON SEXUAL BEHAVIOR AND COMPOSITION OF THE BULL SEMEN}

I 3 bulls were given 30 minutes before the semen collection with an artifical vagina, a subcutaneous injection of either $800 \mathrm{mg}$ of atropine, or of physiological saline. After the injection of atropine the animals show definite reactions of excitement and bloat but their sexual behavior is not disturbed. 
The characteristics of the semen obtained are distinctly modified ; the volume falls from $6, \mathrm{Im}$ to $4,0 \mathrm{ml}$ and the total number from 8,4 billions to 6,6 billions but the concentration rises from I 349 ooo to I 613 ooo spermatozoa $/ \mathrm{mm}^{3}$.

The action of an injection on the composition of the ejaculate is still perceptible after 3 or 4 days, whilst all external reactions have disappeared after 24 hours.

These results could be used for an analysis of the mecanisms of ejaculation.

\section{RÉFÉRENCES BIBLIOGRAPHIQUES}

BLом E., I950. A rapid staining method using eosin-nogrosin to distinguish between live and dead spermatozoa. Nord. vet. Med., 2, 58-6r.

DzIUK P. J., I959. Semen characteristics and sexual behavior in the boar following administration of atropine. J. anim. Sci., 18, I $554^{-1} 555$.

Soulatrac A., Soulairac M. L., i 957. Action de l'amphétamine, de l'adrénaline et de l'atropine sur le comportement sexuel du Rat mâle. J. Physiol., (Paris) 49, $3^{81-3^{85}}$. 